\title{
An Improved Transmission Control Protocol Congestion Control for Satellite Networks
}

\author{
B.R. Han \\ Department of Electrical Engineering \\ Hainan Software Profession Institute \\ Qionghai, China
}

\author{
L. Zong \\ Department of Information Science and Engineering \\ Hunan University of Humanities \\ Science and Technology \\ Loudi, China
}

\begin{abstract}
Transmission control protocol veno is a wireless transmission congestion control algorithm which can better distinguish different loss. It determines whether the packets random loss or congestion loss through calculate to the packets number of backlog in the network. However, transmission control protocol veno does not change the congestion window in the slow start, and it is same to the traditional transmission control protocol. Transmission control protocol adaptive delay and loss response controls the congestion window base on the sender judgments changes in the relevant window and measures round-trip time. It improves the performance of transmission control protocol than the conventional transmission control protocol on the satellite network transmission control protocol. Taking into account the characteristic of both congestion control algorithms respectively, we propose an enhanced transmission control protocol that introduces ideas of transmission control protocol veno distinguish between random packet loss and congestion loss. Our scheme can distinguish the different loss and increase the number of congestion window distinctively in the slow start.
\end{abstract}

Keywords-satellite networks; transmission control protocol congestion; transmission control protocol scheme

\section{INTRODUCTION}

The development of modern computing network gradually transferred from wired network to wireless network, such as the wireless local area network, the Internet of things and the sensor network et al. These wireless networks cannot geographical coverage, such as the voyage of a vessel at sea, military battlefield, remote rural areas. The satellite networks may become the only choice for mobile users at sea to connect with the terrestrial world. However, transmission control protocol performance degrades in geostationary satellite networks due to long propagation delays and high bit error rates. In recent years, many of scholars propose a number of solutions for improve transmission control protocol performance in satellite networks [1-2]. The proposed solutions can be roughly divided into the following three categories: end-to-end improvement solutions [3], link-layer design solutions [4], and transmission control protocol split connection solutions [5-6]. In this paper we consider the satellite network congestion control in maritime environment and propose a modification of transmission control protocol adaptive delay and loss response to improve transmission control protocol performance in broadband geostationary earth orbit satellite network.
The paper is organized as follows. In Section 2, the transmission control protocol adaptive delay and loss response algorithm and transmission control protocol veno are analyzed. In Section 3, our scheme is introduced. Simulation results that compare the performance of transmission control protocol our scheme, transmission control protocol adaptive delay and loss response, transmission control protocol veno and transmission control protocol new reno are presented in Section 4 and conclusions are drawn in Section5.

\section{TRANSMISSION CONTROL PROTOCOL ADAPTIVE DELAY AND LOSS RESPONSE}

Transmission control protocol adaptive delay and loss response (transmission control protocol with algorithm modifications for adaptive delay and loss response) [7] is an end-to-end congestion control algorithm that improves performance for broadband geostationary satellite networks. It introduces division of congestion window increment phase into sub-phases in order to enable transmission of additional segments for better satellite link utilization in the absence of losses. It also adjusts transmission rate more adaptively in the presence of losses. It controls congestion window using the scaling factor $\rho$ which is computed from the measured roundtrip time. It just needs to modify the transmission control protocol sender and divides into three processes: adaptive congestion window (cwnd) increase mechanism, adaptive receives window increase mechanism, and loss recovery mechanism.

Summary, the adaptive cwnd mechanism was proposed to divide the slow start phase into four sub-phases based on the current cwnd, slow start threshold (ssthresh), and flight size (total unacknowledged byte in the network). The cwnd increment factor depends on the sub-phases. In the process of adaptive rwnd increase mechanism, at the transmission control protocol receiver, the rwnd limits the amount of transmitted data. The number of transmitted bytes is the minimum of either the cwnd or rwnd. The adaptive rwnd increase mechanism is used to increment the rwnd when no losses have occurred. Transmission control protocol adaptive delay and loss response allows the transmission of additional segments thus improving bandwidth utilization of a satellite link. The loss recovery mechanism modifies the fast recovery phase to enable the back-to-back transmission of two segments. It limits the number of retransmissions from the retransmission buffer to three segments to prevent a large number of 
unnecessary retransmissions.

The traditional transmission control protocol (transmission control protocol Reno) treats the packet loss as a manifestation of network congestion, and takes appropriate measures to reduce the transmission rate for maintain the stability of the network. It is reliable for the wired network because the bit error rate (bit error rate) is very low in the wired link, such as Twisted-Pair copper wire and fiber optics. Generally, the packet loss can be considered a manifestation of network congestion. However, the reliability of the wireless link is not stable enough in a wireless network environment. This will often results in higher bit error rate. The network may cause randomly packet loss, especially in long delay satellite link which bit error rate can be as high as 10-4. If the network takes the traditional transmission control protocol, such as transmission control protocol Reno, will treat random packet loss as congestion loss, which will results in the transmission control protocol performance degradation. According to the characteristics of the wireless environment, $\mathrm{Fu}$ proposed the transmission control protocol veno [8] that can distinguish effectively between random packet loss and congestion loss in the wireless environment. Transmission control protocol veno is a performance enhancement for transmission control protocol reno in wireless environment. It includes four phases: slow start, congestion avoidance, retransmission timeout, fast retransmit and fast recovery.

The transmission control protocol veno distinguishes between congestion and without congestion in the congestion avoidance phase. It also distinguishes between the random loss and congestion loss in the fast retransmit. The former reduces the oscillation of the transmission control protocol, increases the amount of send data. The latter ensures a high threshold start congestion avoidance when the packet random loss, and also increases the amount of send data. Therefore, based on the judgment of the congestion state, the transmission control protocol veno distinguishes effectively the random loss. In the presence of a large number of random losses of the satellite environment, the transmission rate of transmission control protocol deserves significant improvement.

\section{TRANSMISSION CONTROL PROTOCOL SCHEME}

The transmission control protocol adaptive delay and loss response increases congestion window with different values based on flight size, congestion window and receiver window.

Our transmission control protocol scheme improves mainly three aspects: the congestion window increase, the loss detection and the threshold adjustment. It checks the packet loss in the slow start, congestion avoidance. Because the sender takes the packet loss as the network congestion, the sender's window is set one sender maximum segment size (SMSS). Thus it distinguishes effectively the random loss and the congestion loss, and minimizes the impact of random packet loss for the transmission control protocol performance.

Slow start phase: The transmission control protocol adaptive delay and loss response divided the slow start into four different phases. However, all of the congestion window are used cwnd $=(\sqrt{\rho} / 4) \times$ SMSS in every phase, which does not reflect the difference. In the four sub-phases, our transmission control protocol scheme has a different increase for the congestion window in order to make it adapted to the different growth in the slow start. The slow start begins in the initial connection or the timeout. The sender need detect the network state in the first phase when the initial connection. The value of the congestion window cannot be too high. Our transmission control protocol scheme still uses the transmission control protocol adaptive delay and loss response's settings. The cwnd is $(\sqrt{\rho} / 4) \mathrm{x}$ SMSS. In the second phase and the third phase, the rate of window increase can increase substantially. Our transmission control protocol scheme sets the cwnd is $\sqrt{\rho} \times$ SMSS. The last phase may be appropriate to reduce the rate of window increase, and set one SMSS, as shown in Figure 1.

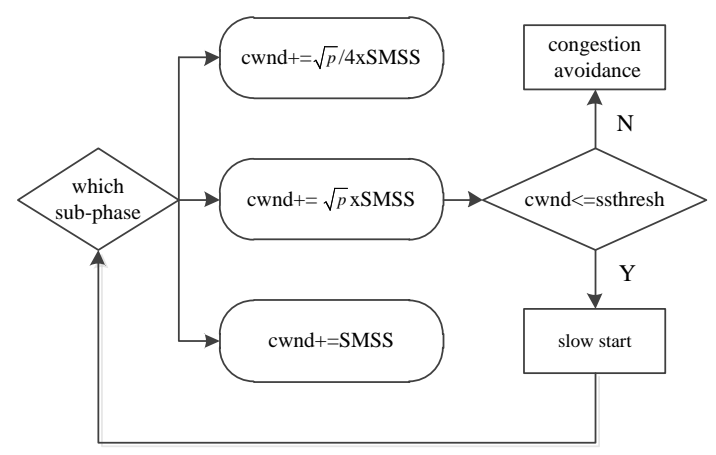

FIGURE I. THREE SUB-PHASES IN THE SLOW START

When a packet loss is detected, if $\mathrm{N} \geq \beta$, the backlog $\mathrm{N}$ at the queue is large, then our scheme treats the packet loss as the congestion loss. The value of congestion window can take the value of the traditional transmission control protocol congestion window. If $\mathrm{N}<\beta$, the backlog $\mathrm{N}$ at the queue is small. Our scheme treats the packet loss as a random loss. The value of the congestion window can take the value of the transmission control protocol veno.

Fast recovery phase: The adaptive delay and loss response also does not consider the distinction between the random loss and the congestion loss when sets the threshold in the fast recovery. It also reduces the window by half as same to the traditional transmission control protocol (new reno). So it also greatly reduces the transmission control protocol performance. Our scheme takes the transmission control protocol veno settings. It distinguishes the loss, and sets the value of threshold is [cwnd $\times 4 / 5$ ] if the packet loss is the random loss, as follows:

\section{// random loss}

if $\mathrm{N}<\beta$ then ssthresh $=\mathrm{cwnd} \times 4 / 5$

//congestion loss

else ssthresh $=\mathrm{cwnd} / 2$

In summary, our proposed transmission control protocol distinguishes the satellite link random loss and adjusts the congestion window and the threshold. It is an enhancement for the transmission control protocol adaptive delay and loss response to improve the transmission control protocol performance in the satellite link. 


\section{SimUlation}

The simulation model is shown in figure 2. The server connects to the gateway. The gateway sends the data to the satellite which connects to the client. The link rate of the server connect to the gateway is set to $10 \mathrm{Mb} / \mathrm{s}$. The downlink and uplink data rates are 2,048 kb / s and $256 \mathrm{~kb} / \mathrm{s}$, respectively. The satellite links one-way propagation delay is $250 \mathrm{~ms}$. The bit error rate of the satellite link sets 10-9, 10-7 and 10-5. The server sends a 50M file size to the client. We evaluate and compare the performance of our proposed transmission control protocol, transmission control protocol adaptive delay and loss response, transmission control protocol veno, and transmission control protocol new reno by collect the client download response time and the satellite link throughput for various bit error rate.

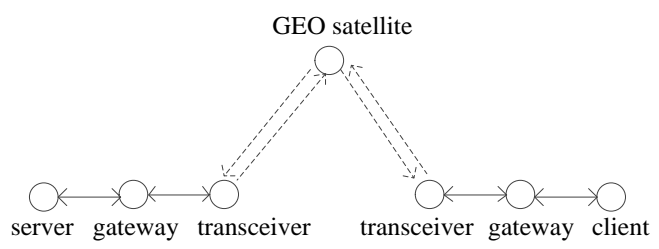

FIGURE II. SIMULATION MODEL

In the case of the gateway without congestion loss, the proposed scheme's download response time has a certain reduce than the other three kinds of transmission control protocol. With the increase of bit error rate, the servicer continuously retransmits the packet that miss and error. Transmission control protocol adaptive delay and loss response and our scheme uses the scale factor of the scaling to increase the window value of the server sends in the slow start or congestion avoidance phase. These can take full advantage of the characteristics of satellite link such as the large bandwidth-delay product. The download response time can greatly reduce than transmission control protocol reno and transmission control protocol veno. Because the slow star phase our proposed scheme differentiate the send window value, it can reduce the download response time.

When a lot of congestion occurs in the network, our proposed scheme has more advantages than other three kinds of transmission control protocol schemes, especially in the case of high bit error rate. The network packet loss come from two aspects, the first is that the gateway cache data loss due to the backlog of large amounts of data in the gateway of the input buffer. There are data losses in the tail sequence. This is the case of the missing data in most of the network. Alternatively in the satellite link, the error bits of data flow caused by high bit error rate, resulting in data link layer the received error bits were discard by receiver. The proposed scheme takes transmission control protocol veno's loss data check in fast recover phase. In the high bit error rate satellite link, the proposed scheme can timely adjust the threshold. In the fast recovery phase the data send rate still maintains a high growth value. These improve the utilization of satellite link.

The proposed scheme's download response time significantly reduce than transmission control protocol adaptive delay and loss response, as show figure 3. Especially the bit error rate changes from 10-6 to 10-5, the download response time rapidly increases. The throughput of satellite link shows in the figure 4 . The proposed scheme also shows a better performance.

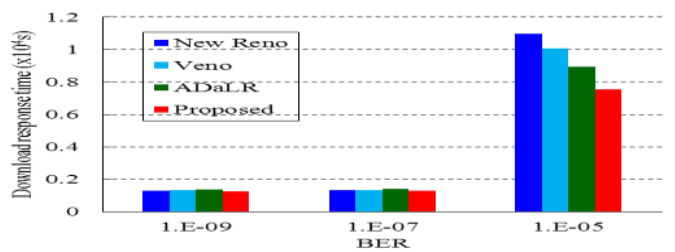

FIGURE III. DOWNLOAD RESPONSE TIME FOR THE SCENARIO WITH CONGESTION LOSS

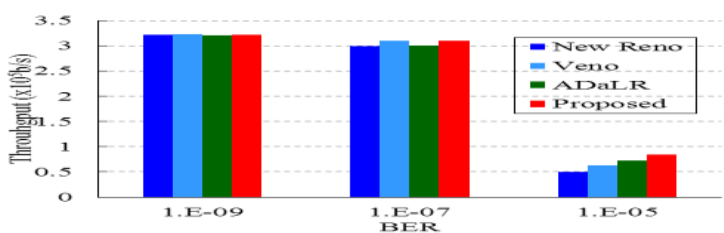

FIGURE IV. SATELLITE LINK THROUGHPUT FOR THE SCENARIO WITH CONGESTION LOSS

\section{CONCLUSION}

In this paper, we propose an enhanced scheme for transmission control protocol adaptive delay and loss response that adapt to the satellite link of the high bit error rate transmission. The proposed scheme checks the packet loss on the satellite link, distinguishes the network congestion packet loss and random packet loss due to bit error, and reasonably adjusts the threshold fast recovery phase, it improves the send rate in the fast recovery, ensures that the growth rate on the satellite link. From the simulation results, the proposed scheme promotes the throughput of satellite link and effectively reduces the file download response time.

\section{ACKNOWLEDGEMENTS}

This work was supported by the Higher School Outstanding Young Backbone Teachers Funded Project of Hainan Province.

\section{REFERENCES}

[1] M. Luglio and M. Y. Sanadidi, M. Gerla, et al, On-board satellite split transmission control protocol proxy. IEEE Journal on Selected Areas in Communications, 22, pp. 362-370, 2004.

[2] A. Pirovano and F. Garcia. A New Survey on Improving Transmission Control Protocol Performances over Geostationary Satellite Link. Network \& Communication Technologies, 1, pp.1-18, 2013.

[3] G. Xylomenos, G. C. Polyzos, P. Mahonen, et al.Transmission control protocol performance issues over wireless link. IEEE Journal on Communications Magazine, 3, pp.52-589, 2001.

[4] T. R. Henderson, H. K Randy and H. Katz. Transport protocols for Internet-compatible satellite networks. IEEE Journal on Selected Areas in Communications, 17, pp.326-344, 1999.

[5] H. Y. Rong and O. K. Victor. Satellite-based internet: a tutorial. IEEE Communications Magazine, 39, pp.154-162, 2001.

[6] Bisio, M. Marchese, and M. Mongelli. Performance Enhanced Proxy Solutions for Satellite Networks: State of the Art, Protocol Stack and Possible Interfaces, Personal Satellite Services. Springer Berlin Heidelberg, 32, pp.61-67, 2009.

[7] M. Omueti and L. Trajković. Transmission control protocol with adaptive delay and loss response for heterogeneous networks in SocialInformatics and Telecommunications Engineering-2007, edited by Peder 
J. Emstad et al., the 3rd International Conference on Wireless internet, Institute for Computer Sciences, Austin, TX, pp. 25-33, 2007.

[8] P. C. Fu and S. C. Liew. Transmission control protocol veno: transmission control protocol enhancement for transmission over wireless access networks. IEEE Journal on Selected Areas in Communications, 2, pp.216-228,2003. 